\title{
Change of Internal Friction on Magnesium Alloy Depending on the Temperature and the Use of Mathematical Methods in the Evaluation of This Property
}

Milan Uhríčik, Andrea Soviarová, Zuzana Dresslerová, Peter Palček, Lenka Kuchariková, Juraj Belan Department of Materials Engineering, Faculty of Mechanical Engineering, University of Žilina, Univerzitná 8215/1, 010 26 Žilina, Slovakia. E-mail: milan.uhricik@fstroj.uniza.sk, andrea.soviarova@fstroj.uniza.sk, zuzana.dresslerova@fstroj.uniza.sk, peter.palcek@fstroj.uniza.sk, lenka.kucharikova@fstroj.uniza.sk,juraj.belan@fstroj.uniza.sk

The article is aimed on present research of internal friction mechanisms that are responsible for the temperature behaviour of AZ91 magnesium alloys. These mechanisms have been studied by ultrasonic resonant apparatus at a frequency close to $20470 \mathrm{~Hz}$ and in a temperature range from $50{ }^{\circ} \mathrm{C}$ up to $400{ }^{\circ} \mathrm{C}$. The specimen on internal friction measurement has an hour glass shape. The specimens were in the as cast state and after measurement showed dendritic structure. Structure of magnesium alloys AZ91 was carried out after the homogenization annealing at temperature $390^{\circ} \mathrm{C}$. It was also used application of mathematical calculations for a more accurately experimental measurement of internal friction depending on the temperature.

Keywords: Internal Friction, Magnesium Alloy, Temperature, Resonant Frequency, Mathematical Calculation

\section{Acknowledgement}

This work has been supported by Scientific Grant Agency of Ministry of Education of Slovak Republic and Slovak Academy of Sciences, No1/0683/15 and project EU ITMS 26110230117.

\section{References}

[1] BLANTER, M. (2007). Internal Friction in Metallic Materials, pp.539, Springer - Verlag: Berlin Heidelberg. ISBN 3-540-68757-2

[2] PUŠKÁR, A.(1995). Vnútorné tlmenie materiálov, pp.382, Žilina: EDIS, ISBN 80-7100-260-7.

[3] SEUNGH, B. (2000). High damping Fe - Mn martensitic alloys for engineering applications, pp. 241-252, Nuclear Engineering and Design, vol.198, issue 3, ISSN 0029-5493.

[4] DRESSLEROVÁ, Z., PALČEK, P. (2014). Temperature dependence of the internal friction measured at different excitation voltages, pp. 287-290, Manufacturing Technology, vol. 14, No. 3, ISSN 1213-2489.

[5] SUGIMOTO, K., MATSUI, K., OKAMOTO, T., KISHITAKE, K. (1975). Effect of Crystal Orientation on Amplitude-Dependent Damping in Magnesium, pp. 647, Trans JIM 16

[6] RIEHEMANN, W. (1998). Internal Friction in Magnesium Material, pp. 61, In B. L. Mordike and K. U Kainer (Eds) Magnesium Alloys and Their Applications, Frankfurt: Werkstoff-Informationsgesellschaft.

[7] HURTALOVÁ, L., TILLOVÁ, E. (2013). Elimination of the negative effect of FE-rich intermetallic phases in secondary (recycled) aluminium cast alloy, pp.44-50. Manufacturing Technology, Vol.13, Num.1, ISSN 12132489.

[8] AVEDESIAN, M., M., BAKER, H. (1999). Magnesium and Magnesium Alloys, pp. 298, Materials Park OH: ASM International, ISBN 0-87170-657-1.

[9] WAN D., WANG J., LIN L., FENG Z., YANG, G. (2008). Damping properties of Mg-Cabinary alloys, pp. 2438 Physical B 403

[10] HLAVÁČOVÁ, I., PALČEK, P., CHALUPOVÁ, M., DRESSLEROVÁ, Z. (2013). Plastic deformation properties of Magnesium alloy AZ61, pp.313-319. Manufacturing Technology, Vol.13, Num.3, ISSN 1213-2489.

[11] DRESSLEROVÁ, Z., PALČEK, P. (2014). Temperature dependence of the internal friction of AZ91 magnesium alloy, pp. 147-148, 31th Danubia-Adria Symposium on advances in experimental mechanics. September 24 - 28 , 2014, Kempten, Germany, ISBN 978-3-00-046740-0

[12] SOVIAROVÁ, A., DRESSLEROVÁ, Z., PALČEK, P., CHALUPOVÁ, M. (2013). Influence of precipitation on internal damping of AZ61 alloy. 30th international colloquium: Visegrád, Hungary - Budapešt', 22-24 May 2013, pp.153-158. ISBN 978-963-313-079-7.

[13] PORUBČAN, J., PALČEK, P., BLAŽEK, D., TROJANOVÁ, Z. (2012). Internal friction in extruded aluminium alloy, pp.197-202. Solid State Phenomena: Internal friction and mechanical spectroscopy. Vol. 184, ISSN 10120394 
[14] SOVIAROVÁ, A., PALČEK, P., BLAŽEK, D. (2013). Analysis of Spurious Effects on Ultrasonic Internal Damping Testing Equipment, In TRANSCOM 2013 10th EUROPEAN CONFERENCE OF YOUNG RESEARCHES AND SCIENTISTS.

[15] KASENČÁK, M. (2010). Vnútorné tlmenie zliatin horčíka v závislosti od amplitúdy deformácie: Dizertačná práca, Žilina: Žilinská Univerzita v Žiline, 97 p.

[16] NAMAŠNÝ, A. (2008). Štúdium vlastností horčíkových zliatin meraním vnútorného tlmenia: Dizertačná práca, Žilina: Žilinská Univerzita v Žiline, 90 p. 